\section{Can Wavelet Denoising Improve Motor Unit Potential Template Estimation?}

\author{
Hasanzadeh S. H. ${ }^{\oplus}$, Parsaei H. ${ }^{2,3 * \odot}$, Movahedi M. M. ${ }^{2}$
}

\begin{abstract}
Background: Electromyographic (EMG) signals obtained from a contracted muscle contain valuable information on its activity and health status. Much of this information lies in motor unit potentials (MUPs) of its motor units (MUs), collected during the muscle contraction. Hence, accurate estimation of a MUP template for each MU is crucial.
\end{abstract}

Objective: To investigate the possibility of improving MUP template estimation using the wavelet denoising technique.

Material and Methods: In this analytical study, several MUP template estimators were developed by combining conventional estimation methods and wavelet denoising techniques. A MUP template was initially estimated using conventional methods such as mean, median, median-trimmed mean, or mode. Thereafter, it was post-processed using the wavelet denoising technique.

Results: Evaluation results of the studied estimators using 40 simulated EMG signals with a true template for each constituent MUP train showed that augmented wavelet- based template estimation methods are more reliable than conventional methods. However, on average, wavelet denoising was not much effective. Around 40 MUPs of a MU is sufficient to estimate its MUP template.

Conclusion: Although wavelet techniques are effective in EMG signal analysis, here wavelet denoising did not practically improve MUP template estimation. Considering computational simplicity and estimation error, the two methods median and median-trimmed mean are practical estimators that can provide a good estimation of a MUP template for a MU when approximately 40 MUPs are available. Nevertheless, the baseline noise level in the MUP templates estimated using the median-trimmed mean method is slightly lower than that in the templates estimated using the median method.

Citation: Hasanzadeh SH. Parsaei H. Movahedi MM. Can Wavelet Denoising Improve Motor Unit Potential Template Estimation? J Biomed Phys Eng. 2020;10(2):197-204. doi: 10.31661/jbpe.v0i0.2001-1043.

\section{Keywords}

Electromyography; EMG; MUP Template Estimation; Signal Processing; Wavelet Analysis

\section{Introduction}

$\Omega$ here are numerous $(>100)$ neuromuscular and neurological disorders that affect the spinal cord, nerves, or muscles. These disorders change the morphology and activation patterns of the motor units (MUs) of the involved muscle. Therefore, the characteristics of electromyographic (EMG) signals detected from a muscle reflect the degree and type of muscle disorder [1-3]. Early detection and diagno-
${ }^{1} \mathrm{MSc}$, Department of Medical Physics and Engineering, Shiraz University of Medical Sciences, Shiraz, Iran ${ }^{2} \mathrm{PhD}$, Department of Medical Physics and Engineering, Shiraz University of Medical Sciences, Shiraz, Iran

${ }^{3} \mathrm{PhD}$, Shiraz Neuroscience Research Center, Shiraz University of Medical Sciences, Shiraz, Iran

*Corresponding author: H. Parsaei

Department of Medical

Physics and Engineer-

ing, Shiraz University

of Medical Sciences,

Shiraz, Iran

E-mail: hparsaei@sums. ac.ir

Received: 4 January 2020 Accepted: 26 January 2020 
Hasanzadeh S. H., Parsaei H., Movahedi M. M.

sis of these diseases are essential for treatment and management. One way to detect these diseases is by electromyography, which is the technique of detecting, processing and analyzing EMG signals.

During conventional EMG examination, a clinician visually or auditory assesses the characteristics of EMG signals detected using needle electrodes positioned at various locations within a muscle to form an overall impression of its condition. Such a subjective assessment is highly dependent on the skills and experience level of the clinician and is prone to high error rate and operator bias. Quantitative electromyography (QEMG) methods have been developed to overcome these issues [4-8]. QEMG methods characterize motor unit potential (MUP) waveforms automatically using pattern recognition techniques, allowing for greater objectivity and reproducibility in supporting the diagnostic process [4, 9-11]. However, the efficacy of QEMG methods is directly connected to the accuracy with which MUP waveforms can be estimated.

The QEMG process entails decomposing an acquired EMG signal into constituting motor unit potential trains(MUPTs), estimating MUP templates and firing pattern statistics of the MUs that contributed to EMG signal, extracting features of MUP templates, and finally analyzing the extracted features $[4,6]$. A MUP template for a MU is a MUP waveform that represents the shape of the potentials generated by the MU. This template is estimated by combining all the MUPs within the MUPT, produced by the MU. Due to background noise, interference from other MUs, and biological MUP shape variability of the MU (mainly because of jitter), estimating the MUP template is subject to some errors. Several methods, such as mean, median, median trimmed mean, statistical and interference canceling averaging techniques have been proposed to reduce the effect of interference to provide reliable MUP template estimates for individual MU [12-16]. These methods have produced prom- ising results and performance, but their accuracy decreases significantly when the number of MUP samples extracted for a specific MU is small. This can lead to uncertainty in the information provided by QEMG. Therefore, developing an efficient algorithm to estimate a MUP template is of interest.

Wavelet transforms have been widely used for biomedical signal processing as signal analysis, signal denoising, and signal compression [17-19]. In EMG signal analysis, these techniques have shown great promises in extracting MUP features in EMG signal decomposition and muscle characterization [20-23]. In this work, we investigated the effectiveness of the wavelet denoising technique as a post-processing practice to improve the estimation of the MUP template.

\section{Material and Methods}

This analytical study includes two main steps: data collection, and MUP template estimation. In data collection step, several MUPTs with known MUP template were collected. In MUP template estimation step, various MUP template estimation methods were applied to estimate the MUP template of a MU using its MUPs in the corresponding train. Details of each step are provided in the following two sub-sections.

\section{Data Collection}

Simulated EMG signals were used to examine the performance of the algorithms developed/studied in this work. Specifically, 40 EMG signals were simulated using a physiologically-based EMG simulator that can generate physiologically realistic EMG signals [24]. We used simulated EMG signals because the employed simulator provides gold standard (true) MUP templates for MUPTs of a generated EMG signal. This assisted in the comparison of the estimated MUP template with the exact template, which increased certainty in the evaluation of the utilized algorithms. In addition, the simulator provides the capability 
Wavelet Denoising in MUP Template Estimation

of including several artifacts affecting the accuracy in MUP template estimation.

The simulation was done with various parameters: neuromuscular transmission variability (jitter) was set as 0 (zero), 25, or 50 $\mu \mathrm{s}$; the number of MU varied from 3 to 12 , and maximum voluntary contraction (MVC) was considered at two levels $5 \%$ or $10 \%$. Each signal was decomposed, using DQEMG software to extract constituent MUTPs. An example MUPT of the dataset used in this study is shown in Figure 1. The simulated signal length was 30 -second and the sampling rate was $31.25 \mathrm{kHz}$.

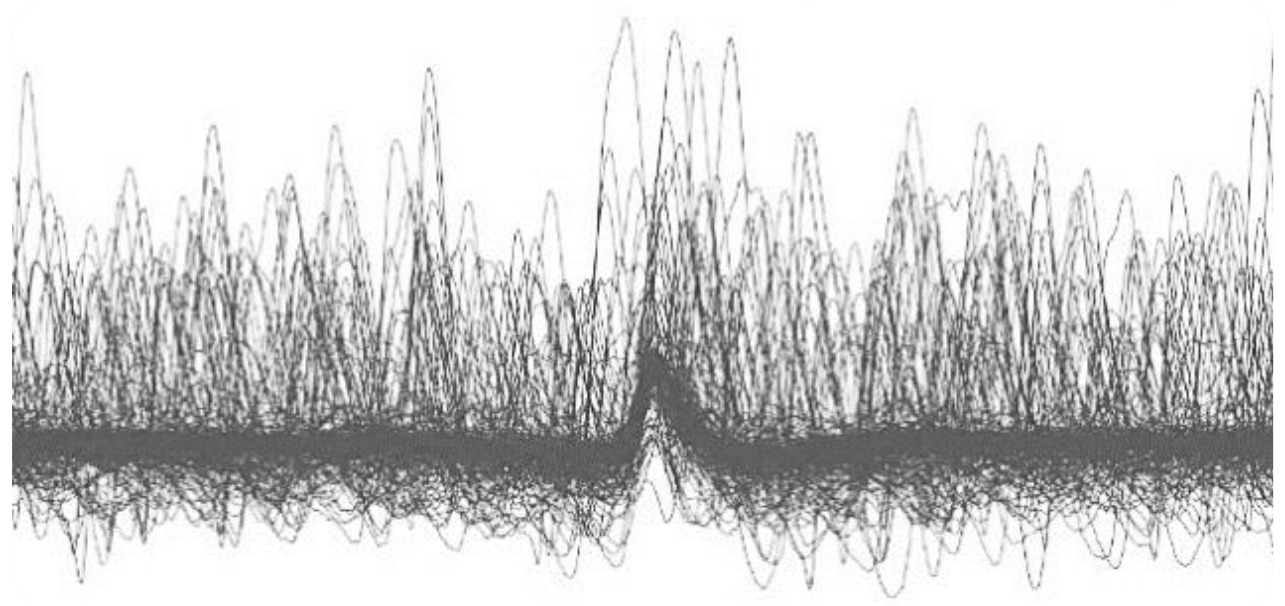

Figure 1: An example of a motor unit potential train used in this study.

To study the effect of sample size (here the number of MUPs) in the estimation of the MUP template, around 700 MUPTs with 20 to 300 MUPs were generated by randomly selecting MUPs of the MUTPs obtained from decomposing the signals.

\section{MUP Template Estimation}

Assuming $X_{d \times n}$ represents a MUPT containing $d$ MUPs, each of which represented by $n$ time samples of a MUP, the problem of MUP template estimation is to use the information provided in this matrix for estimating an $n$-dimensional vector that can accurately represent MUP waveform shape of the MU that generated this MUPT. In this work, for a given MUPT, a MUP template was initially estimated, using conventional methods including mean, median, median-trimmed mean, or mode [14-16]. Thereafter, the primary estimated template was post-processed using the wavelet denois- ing technique.

In using wavelet-based signal denoising techniques, several parameters including the type of mother wavelet, the thresholding techniques, and the desired number of resolution level have to be addressed. Here, we chose the mother wavelet using the cross-validation strategy. Specifically, we investigated forty mother wavelet functions from orthogonal and biorthogonal families including Daubechies (db1-db10), Symlets (sym1-sym20), Coiflets (coif1-coif5), Biorthogonal (1.1-6.8), dMeyer, and Haar. The mother wavelet provided the overall highest similarity value with the initial MUP templates and the lowest recovery error measured by the mean squared error was chosen as the best mother wavelet. The similarity and compatibility of the mother wavelet with the MUPs show that the wavelet can characterize the signal well. In terms of the thresholding technique, we used the soft threshold- 
Hasanzadeh S. H., Parsaei H., Movahedi M. M.

ing method to preserve the details of the MUP template. Ultimately, "Coiflet5" from Coiflets family revealed to have the best performance.

\section{Results}

The performance of the studied MUP template estimators was quantitatively evaluated by comparing the estimated MUP templates with the true ones provided by the simulator. Three indices mean squared error (MSE), signal-to-noise ratio (SNR), and RMS ratio were used as performance measures. The MSE was estimated by calculating the error between the original template and its estimated version. This common estimation performance measure is mostly used due to its computational simplicity, which causes this measure to be easy to understand and analyze. The RMS ratio index is defined as the ratio of the RMS of the estimated template divided by that of the true template. Evidently, the closer the value of RMS ratio to one, the better the model is in reconstructing the information in the template.

Figures 2, 3 and 4 depict the performance of the examined MUP template estimators for the three indices MSE, SNR, and RMS ratio versus the number of MUPs available for a MU in the corresponding MUPT. As shown, template estimation improves as the number of MUPs increases. This is in line with the concept of sample mean estimation, where the standard error reduces as the square root of the number of averaged samples. Based on the obtained results, the two methods median and median-

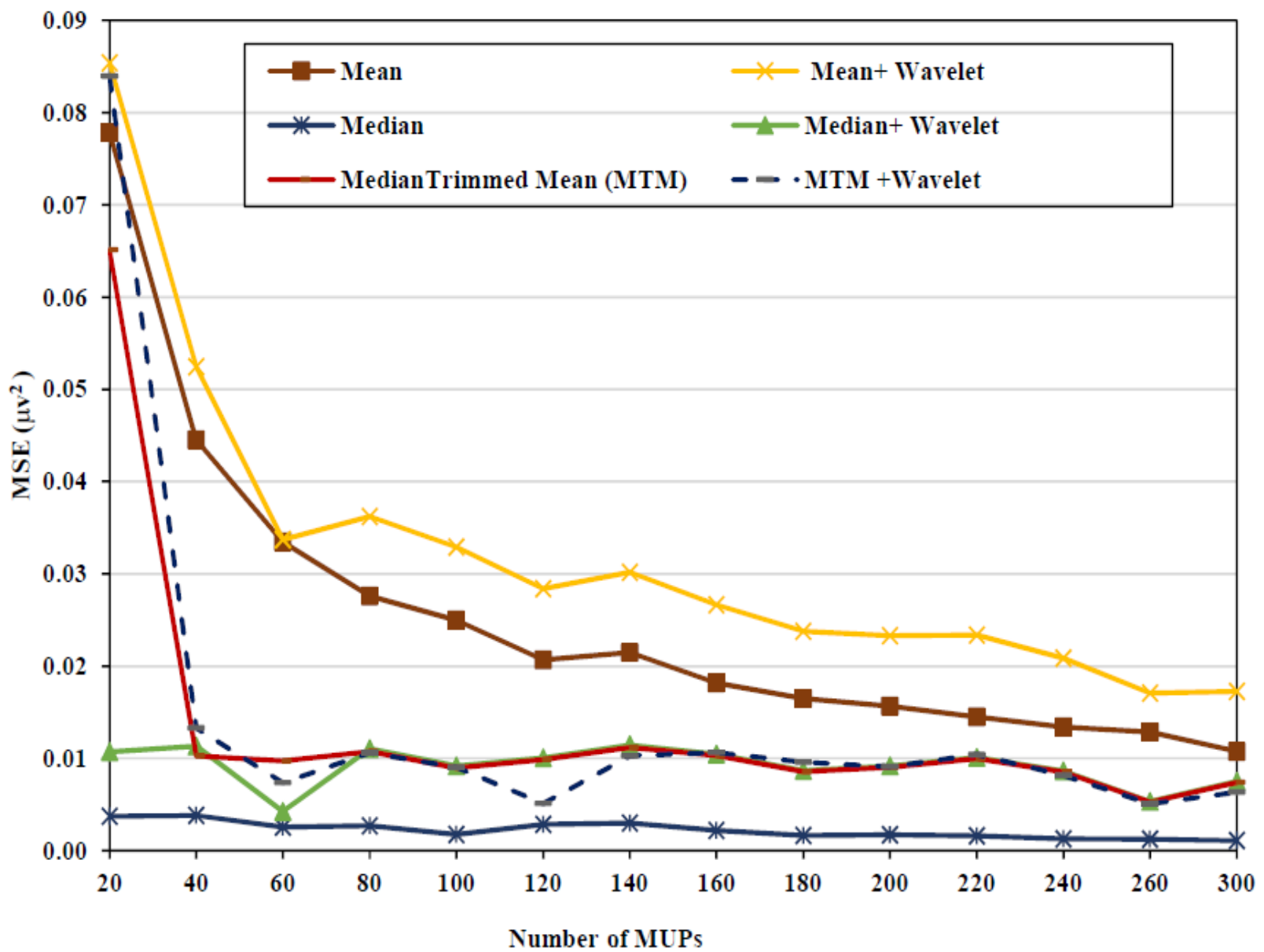

Figure 2: Mean square error in estimating a MUP template versus the number of MUPs available for the motor unit. 


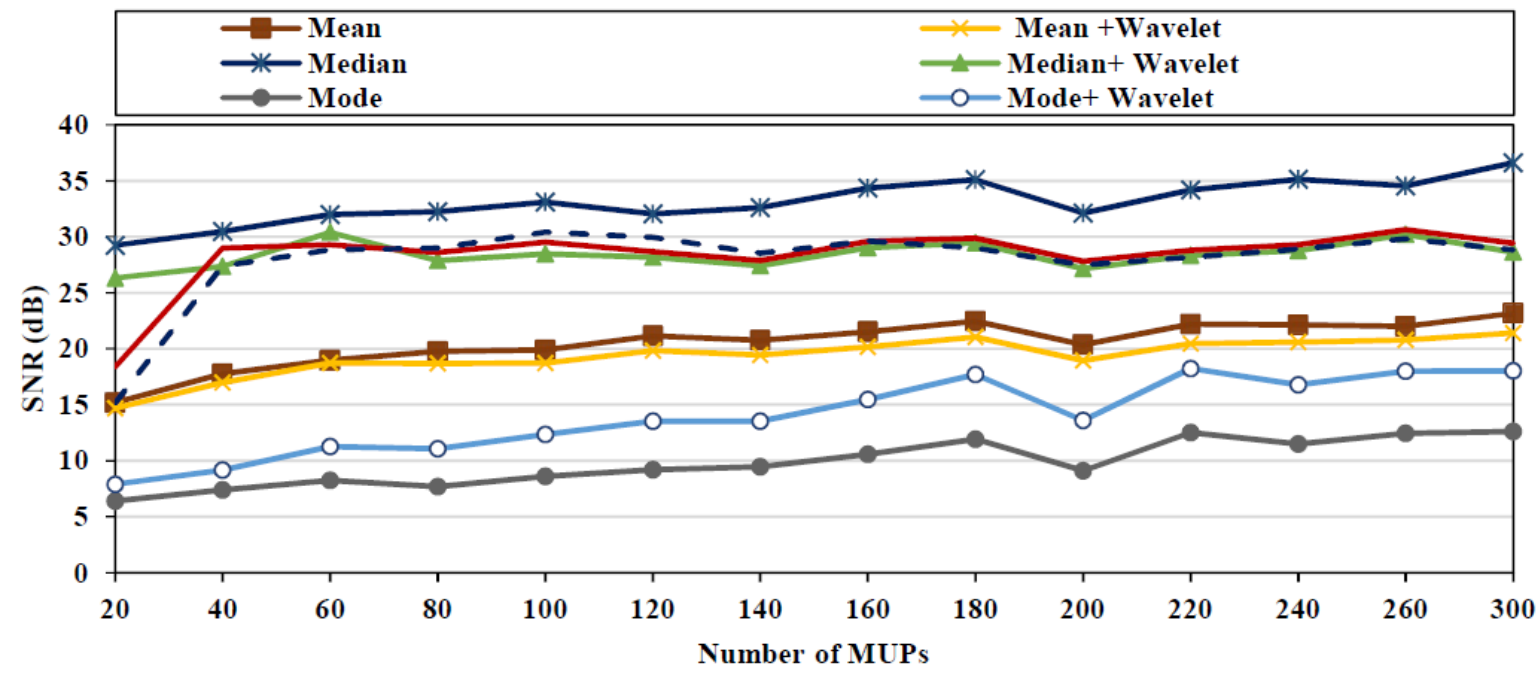

Figure 3: Signal to noise ratio of a MUP template estimated for a motor unit versus the number of MUPs available for the motor unit.

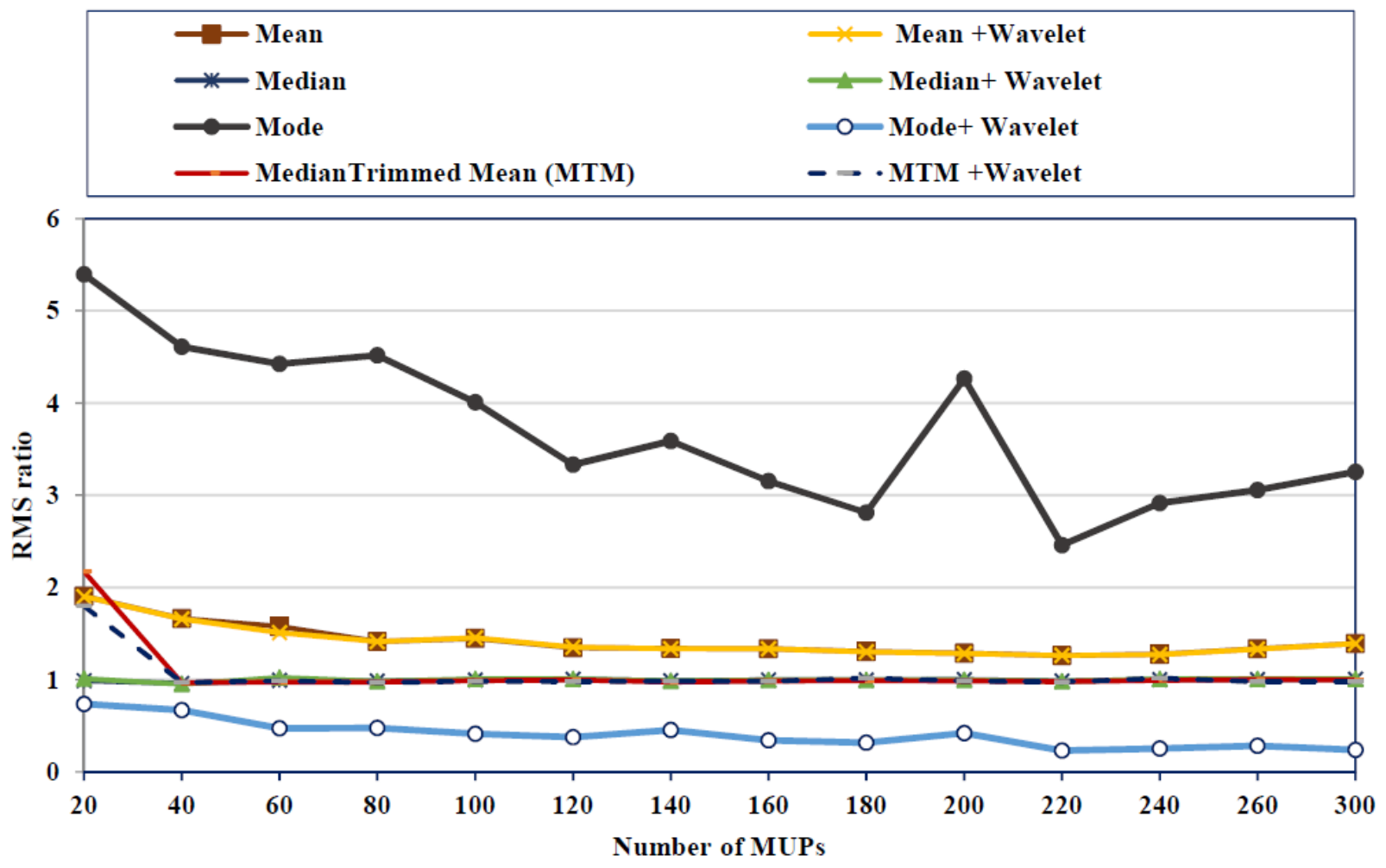

Figure 4: The ratio of the RMS of an estimated MUP template over that of the true template (RMS ratio) versus the number of MUPs available for a motor unit in the corresponding motor unit potential train. 
Hasanzadeh S. H., Parsaei H., Movahedi M. M.

trimmed mean are the two top estimators.

\section{Discussion}

Accurate estimation of a MUP template for a MU that precisely represents the shape of the potentials generated by the MU is a key factor in QEMG. In this work, we examined several methods for this task. Based on the obtained results (Figures, 2,3, and 4), the two medianbased methods median and median- trimmed mean are the two top estimators. This finding is consistent with previous studies that showed these two methods outperformed other MUP template estimation methods $[14,15]$.

The results in Figures 2, 3 and 4 show that there is an elbow in the curves of the performance measures when the number of MUPs $>40$. This shows that 40 MUPs of a motor unit are sufficient to estimate a MUP template, representing MUP waveform shape generated by the MU. However, statistical comparison of the variances of the performance indices over various level of the number of MUPs showed that when the number of MUPs increases the reliability of the estimator increases as well, except for the two methods median, and median-trimmed mean that show almost constant variability in performance measure over various number of MUPs in MUPTs.

In terms of the effectiveness of wavelet denoising, this technique assisted with improving SNR of the MUP templates initially estimated using the Mode estimator. However, for the other estimators, wavelet denoising was not practically effective in noise reduction. The value of SNR could be improved further by increasing the value of the threshold in wavelet denoising; however, this leads to a reduction in the amplitude of the template. Wavelet techniques have been shown to be effective in EMG signal analysis, such as extracting MUP features in EMG signal decomposition and muscle characterization [20-23], but this technique was not that effective in MUP template estimation. Nevertheless, pair- wise statistical comparison of the variances of the performance indices (conventional method versus corresponding augmented wavelet) showed that augmented wavelet denoising template estimation methods are more reliable (less variable) than the conventional methods.

In this experiment, we used MUPTs that include several unresolved superimposed MUPs, since DQEMG software does not resolve superimposed MUPs. Therefore, the studied MUP template estimators might lead to better results, if applied to MUPTs extracted by an EMG decomposition software that can resolve superimposed MUPs.

As with any experimental work, there were several limitations with this research. The methods were evaluated using only simulated EMG signals. We used simulated EMG signals because the software used for generating EMG signals provides a true MUP template for each MUPT of a generated EMG signal. This assisted in the comparison of the estimated MUP template with the exact template, which increased certainty in the evaluation of the utilized algorithms. However, there are several limitations with the EMG simulator used; for example, the issue of needle sliding during signal recording cannot be included in the simulator. Therefore, the data used in this paper might not exactly fit with real clinical data. Nevertheless, several key artifacts that affect the estimation of MUP template (e.g., MUP shape variability) were included in creating the data set. Consequently, the same results are expected for the real signals.

\section{Conclusion}

Motor unit potential templates of motor units that were active during an EMG signal recording can provide valuable diagnostic information. Several artifacts, such as jitter, interference from MUPs generated from other MUs, and equipment noise affect the estimation of a MUP template. Therefore, reducing the effects of these artifacts to improve MUP template estimation is of interest. In this pa- 
Wavelet Denoising in MUP Template Estimation

per, we investigated the effectiveness of the wavelet denoising method to reach this objective. The obtained results showed that augmented wavelet denoising template estimation methods are more reliable (less variable) than conventional methods. However, on average wavelet denoising was not practically effective. The obtained results revealed that the median-trimmed mean estimator is the best MUP template estimator. Finally, the results showed that 40 MUPs of a motor unit is sufficient to estimate its MUP template.

\section{Acknowledgment}

The paper has been extracted from parts of the M.Sc. thesis by Hassan Hassanzahed supported by the Research Council of Shiraz University of Medical Sciences under Grant number 94-7450. The authors wish to thank Mr. H. Argasi at the Research Consultation Center (RCC) of Shiraz University of Medical Sciences for his invaluable assistance in editing this manuscript.

\section{Conflict of Interest}

\section{None}

\section{References}

1. Rubin D, Daube J. Clinical Neurophysiology. 4th edition. Oxford, New York: Oxford University Press; 2016.

2. Lipa BM, Han JJ. Electrodiagnosis in Neuromuscular Disease. Phys Med Rehabil Clin N Am. 2012;23:565-87. doi: 10.1016/j. pmr.2012.06.007. PubMed PMID: 22938876.

3. Aminoff MJ. Electromyography in Clinical Practice: Clinical and Electrodiagnostic Aspects of Neuromuscular Disease. 3rd edition. New York: Churchill Livingstone; 1997.

4. Farkas $C$, Hamilton-Wright A, Parsaei $H$, Stashuk DW. A review of clinical quantitative electromyography. Crit Rev Biomed Eng. 2010;38:46785. doi: 10.1615/critrevbiomedeng.v38.i5.30. PubMed PMID: 21175409.

5. Podnar S, Voduscaronek DB, Stålberg E. Comparison of quantitative techniques in anal sphincter electromyography. Muscle Nerve. 2002;25:8392. doi: $10.1002 / m u s .10017$. PubMed PMID: 11754189 .
6. Stashuk DW. Decomposition and quantitative analysis of clinical electromyographic signals. Med Eng Phys. 1999;21:389-404. doi: 10.1016/ s1350-4533(99)00064-8. PubMed PMID: 10624736.

7. Stålberg E, Nandedkar SD, Sanders DB, Falck B. Quantitative Motor Unit Potential Analysis. Clin Neurophysiol. 1996;13:401-22. doi: 10.1097/00004691-199609000-00004. PubMed PMID: 8897206

8. Yu YL, Murray NMF. A comparison of concentric needle electromyography, quantitative EMG and single fibre EMG in the diagnosis of neuromuscular diseases. Electroencephalogr Clin Neurophysiol. 1984;58:220-5. doi: 10.1016/00134694(84)90107-x. PubMed PMID: 6205853.

9. Kamali T, Stashuk DW. Electrophysiological Muscle Classification Using Multiple Instance Learning and Unsupervised Time and Spectral Domain Analysis. IEEE Trans Biomed Eng. 2018;65:2494502. doi: 10.1109/TBME.2018.2802200. PubMed PMID: 29993485.

10. Kamali T, Stashuk DW. A Density-Based Clustering Approach to Motor Unit Potential Characterizations to Support Diagnosis of Neuromuscular Disorders. IEEE Trans Neural Syst Rehabil Eng. 2017;25:956-66. doi: 10.1109/TNSRE.2017.2673664. PubMed PMID: 28252410.

11. Yousefi J, Hamilton-Wright A. Characterizing EMG data using machine-learning tools. Comput Biol Med. 2014;51:1-13. doi: 10.1016/j.compbiomed.2014.04.018. PubMed PMID: 24857941.

12. Hamilton-Wright A, Stashuk DW. Improved MUP Template Estimation Using Local Time Warping and Kernel Weighted Averaging. Conf Proc IEEE Eng Med Biol Soc. 2018;2018:2647-50. doi: 10.1109/EMBC.2018.8512886. PubMed PMID: 30440951.

13. Malanda-Trigueros A, Navallas J, Rodriguez-Falces J, Rodriguez-Carreño I, Porta S, FernándezMartínez $\mathrm{M}$, et al. Sliding window averaging in normal and pathological motor unit action potential trains. Clin Neurophysiol Off J Int Fed Clin Neurophysiol. 2018;129:1170-81. doi: 10.1016/j. clinph.2018.02.134. PubMed PMID: 29649769.

14. Malanda A, Navallas J, Rodriguez-Falces J, Rodriguez-Carreño I, Gila L. Averaging methods for extracting representative waveforms from motor unit action potential trains. J Electromyogr Kinesiol. 2015;25:581-95. doi: 10.1016/j.jelekin.2015.04.007. PubMed PMID: 25962870.

15. Stashuk DW. Mean, median and mode estimation of motor unit action potential templates. Proc 
Hasanzadeh S. H., Parsaei H., Movahedi M. M.

18th Annu Int Conf IEEE Eng Med Biol Soc. Amsterdam: IEEE; 1996(4). p. 1498-9. doi: 10.1109/ IEMBS.1996.647521.

16. Nandedkar SD, Sanders DB. Median averaging of electromyographic motor unit action potentials: comparison with other techniques. Med Biol Eng Comput. 1989;27:566-71. doi: 10.1007/ BF02441637.

17. Rafiee J, Rafiee MA, Prause N, Schoen MP. Wavelet basis functions in biomedical signal processing. Expert Syst Appl. 2011;38:6190-201. doi: 10.1016/j.eswa.2010.11.050.

18. Akay M, editor. Time Frequency and Wavelets in Biomedical Signal Processing. 1st Edition. Piscataway, NJ: Wiley-IEEE Press; 1997.

19. Akay M. Wavelets in biomedical engineering. Ann Biomed Eng. 1995;23:531-42. doi: 10.1007/ bf02584453. PubMed PMID: 7503456.

20. Ghofrani Jahromi M, Parsaei H, Zamani A, Stashuk DW. Cross Comparison of Motor Unit Potential Features Used in EMG Signal Decomposition. IEEE Trans Neural Syst Rehabil Eng. 2018;26:101725. doi: 10.1109/TNSRE.2018.2817498. PubMed
PMID: 29752237.

21. Ghofrani Jahromi M, Parsaei $H$, Zamani A, Dehbozorgi M. Comparative Analysis of Waveletbased Feature Extraction for Intramuscular EMG Signal Decomposition. J Biomed Phys Eng. 2017;7:365-78. PubMed PMID: 29392120 PMCID: PMC5758715.

22. Zennaro D, Wellig P, Koch VM, Moschytz GS, Laubli T. A software package for the decomposition of long-term multichannel EMG signals using wavelet coefficients. IEEE Trans Biomed Eng. 2003;50:58-69. doi: 10.1109/ TBME.2002.807321. PubMed PMID: 12617525.

23. Ren X, Hu X, Wang Z, Yan Z. MUAP extraction and classification based on wavelet transform and ICA for EMG decomposition. Med Biol Eng Comput. 2006;44:371-82. doi: 10.1007/s11517006-0051-3. PubMed PMID: 16937179.

24. Hamilton-Wright A, Stashuk DW. Physiologically based simulation of clinical EMG signals. IEEE Trans Biomed Eng. 2005;52:171-83. doi: 10.1109/TBME.2004.840501. PubMed PMID: 15709654. 